\title{
Solving heat transfer in a timber beam exposed to fire with methods based on radial basis functions (RBFs)
}

\author{
L.Vrankar ${ }^{1,4}$, G. Turk ${ }^{2}$, E. J. Kansa ${ }^{3}$ \& F. Runovc ${ }^{4}$ \\ ${ }^{1}$ Slovenian Nuclear Safety Administration, Slovenia \\ ${ }^{2}$ University of Ljubljana, FGG, Slovenia \\ ${ }^{3}$ Department of Mechanical and Aeronautical Engineering, \\ University of California, USA \\ ${ }_{4}^{4}$ University of Ljubljana, NTF, Slovenia
}

\begin{abstract}
The objective of this paper is to represent an alternative approach to conventional numerical methods for solving heat transfer and charring behaviour of timber when exposed to fire. The model consists of differential equations for heat transfer with the corresponding boundary conditions. The char formation in the wood beam as a function of its temperature is also taken into account by the model. Picard's or Newton's methods are used for solving the second-order non-linear partial differential equations. In recent years, the RBF methods have emerged as novel computing methods in the scientific computing community. Traditionally, the most popular methods have been the finite element methods (FEM), the finite difference methods (FDM), and boundary element method (BEM). The results are tested on the one-dimensional case in standard fire conditions, for which the comparison is made with the results of one-dimensional charring rate models for wood. The same model is used to analyze a two-dimensional behaviour of a timber beam exposed to fire from three sides.

Keywords: heat transfer, charring behaviour of timber, pseudospectral mode, multiquadric, partial differential equations, Picard's method, radiation, pyrolysis, combustion.
\end{abstract}




\section{Introduction}

When wood is exposed to fire it undergoes thermal degradation. Thermal degradation or pyrolysis reduces the density by changing the wood to char and gases. The pyrolysis gases undergo flaming combustion as they leave the charred wood surface. The pyrolysis, charring, and combustion of wood have been extensively studied in [1].

The governing equations present differential equations for heat transfer with the corresponding boundary conditions. The boundary conditions prescribe the heat flow on the exposed boundaries of cross-section. In our case, different types of boundary conditions were used. The char formation in the wood beam as a function of its temperature is also taken into account by the model. The problem is solved numerically by the radial base function $(\mathrm{RBF})$ methods.

Recent research on the numerical method has focused on the idea of using a meshless methodology for the numerical solution of PDEs. One of the common characteristics of all mesh-free methods is their ability to construct functional approximation or interpolation entirely from information at a set of scattered nodes, among which no relationship or connectivity is needed. In this paper, two methods will be used, Kansa's approach [2] and pseudospectral (PS) method [3,4]. The goal of this paper is to represent an alternative approach to conventional numerical methods for solving heat transfer and charring behaviour of timber when exposed to fire.

The results are tested on the one dimensional case in standard fire conditions, for which the comparison is made with the results of one-dimensional charring rate models for wood presented in the literature published by White and Nordheim [5]. The same model is used to analyse a two-dimensional behaviour of wood beam exposed to fire from three sides. The results are compared with the results obtained in literature [6]. Faster charring at the corners and typical rounding effect are observed.

\section{Governing equations}

In general, the heat and mass transfer is governed by the two second order non-linear partial differential equations [7]. In our case, only equation which describes heat conduction governed predominantly by temperature gradients was considered. The equation can be written as:

$$
\varrho c_{p} \frac{\partial T}{\partial t}=k_{x} \frac{\partial^{2} T}{\partial x^{2}}+k_{y} \frac{\partial^{2} T}{\partial y^{2}},
$$

where $k_{x}$ and $k_{y}$ represent thermal conductivity $(\mathrm{W} / \mathrm{mK})$ in directions $x$ and $y$ of a cross-section of the beam, $\varrho$ is density $\left(\mathrm{kg} / \mathrm{m}^{3}\right), c_{p}$ specific heat $(\mathrm{J} / \mathrm{kgK})$ and $T$ temperature $\left({ }^{\circ} \mathrm{C}\right)$. The second equation describes moisture diffusion governed by moisture potential and is not considered here.

The problem is complete when initial and boundary conditions are specified. The initial condition prescribes the temperature in the cross-section of the beam at 
the initial time $t=0$

$$
T(x, y, 0)=T_{0}(x, y) .
$$

The boundary conditions prescribe the heat flow on the exposed boundaries of cross-section. Thus, the boundary conditions at the exposed surface are given by balancing heat conduction at the surface with the radiative and convective heat flux. The boundary conditions can be written as:

$$
-k_{x} \frac{\partial T}{\partial x} e_{n x}-k_{y} \frac{\partial T}{\partial y} e_{n y}=h_{c}\left(T-T_{A}\right)+\varepsilon_{R} \sigma\left(T^{4}-T_{R}^{4}\right),
$$

where $e_{n x}$ and $e_{n y}$ are components of the normal to the boundary surface and $h_{c}$ is convective heat transfer coefficient $\left(\mathrm{W} / \mathrm{m}^{2} \mathrm{~K}\right) . T_{A}$ is the temperature of the ambient. $T_{R}$ is the temperature of the radiating surface, $\varepsilon_{R}$ is the effective surface emissivity of the exterior siding and $\sigma$ is the Stephan-Boltzmann constant for radiation, $\left(\sigma=5 \cdot 671 \cdot 10^{-8} \mathrm{~W} / \mathrm{m}^{2} \mathrm{~K}^{4}\right)$.

\section{Solving the heat conduction equation}

For the solution of eqn. (1) with the corresponding initial and boundary conditions non-symmetric RBF and PS method are used. A computer program is written in $M A T L A B$ environment.

\subsection{Kansa's approach}

A very popular non-symmetric method for the solution of PDEs with RBFs was suggested by Kansa [2]. A radial basis function is the function $\varphi_{j}(\mathbf{x}):=$ $\varphi\left(\left\|\mathbf{x}-\mathbf{x}_{j}\right\|\right)$, which depends only on the distance between $\mathbf{x} \in \mathbb{R}^{d}$ and a fixed point $\mathbf{x}_{j} \in \mathbb{R}^{d}$. Here, $\varphi_{j}$ is continuous and bounded on any bounded sub-domain $\Omega \subseteq \mathbb{R}^{d}$ whereas $\varphi: \mathbb{R}^{d} \rightarrow \mathbb{R}$. Let $r \geq 0$ denote the Euclidean distance between any pair of points in the domain $\Omega$. The commonly used radial basis functions are linear $(\varphi(r)=r)$, cubic $\left(\varphi(r)=r^{3}\right)$, thin-plate spline $\left(\varphi(r)=r^{2} \log r\right)$ and Gaussian $\left(\varphi(r)=e^{-\alpha r^{2}}\right)$. The most popular globally supported $C^{\infty} \mathrm{RBFs}$ are multiquadric functions (MQ) $\left(\varphi(r)=\left(1+(r / c)^{2}\right)^{\beta}\right), \beta=1 / 2$ [8]. MQ has been already efficiently used in transport problems [9], moving-boundary problems [10], etc.

The starting point of the RBFs solution of partial differential equations is the interpolation problem. The MQ RBFs is used to interpolate the scalar level set function by using MQ basis centered at these $R B F$ centers, $\Xi=\left\{\xi_{1}, \ldots, \xi_{\mathbf{N}}\right\}$. The goal is to find an interpolant of the form

$$
\Phi(\mathbf{x})=\sum_{j=1}^{N} \alpha_{j} \varphi\left(\left\|\mathbf{x}-\xi_{j}\right\|\right), \quad \text { such that } \Phi\left(\mathbf{x}_{i}\right)=f_{i}, i=1, \ldots, N,
$$

where $\alpha_{j}$ is the weight of the radial basis function positioned at the $j$-th center. Knowing that the initial data values $f_{1}, \ldots, f_{N} \in \mathbb{R}$ at the data points 
$\mathbf{x}=\left\{\mathbf{x}_{1}, \ldots, \mathbf{x}_{N}\right\} \subset \Omega \subset \mathbb{R}^{N}$, the solution of this problem leads to a linear system $\mathbf{A} \boldsymbol{\alpha}=\mathbf{f}$ with the entries of $\mathbf{A}$ given by

$$
A_{i j}=\varphi\left(\left\|\mathbf{x}_{i}-\xi_{j}\right\|\right), \quad i, j=1, \ldots, N \text {. }
$$

We now switch to the collocation solution of partial differential equations. We consider a PDE in the general form of

$$
\begin{gathered}
\mathrm{L} u(\mathbf{x})=f(\mathbf{x}), \quad \text { in } \Omega \subset \mathbb{R}^{d}, \\
\mathrm{~B} u(\mathbf{x})=g(\mathbf{x}), \text { on } \partial \Omega,
\end{gathered}
$$

where $u$ is the unknown solution, $d$ denotes the dimension, $\partial \Omega$ is the boundary of the domain $\Omega, \mathrm{L}$ is the differential operator on the interior, and $\mathrm{B}$ is the operator that specifies the boundary conditions of the Dirichlet, Neumann or mixed type. $f(\mathbf{x})$ and $g(\mathbf{x})$ are given functions with sufficient smoothness mapping $\mathbb{R}^{d} \mapsto \mathbb{R}$, respectively.

The unknown PDE solution $u$ is approximated by RBFs in the form:

$$
u \approx U(\mathbf{x})=\sum_{j=1}^{N} \alpha_{j} \varphi\left(\left\|\mathbf{x}-\xi_{j}\right\|\right) .
$$

The collocation matrix that arises when matching the eqn. (6) and eqn. (7) at the collocation points $\Xi$ will be of the form

$$
\mathbf{A}=\left[\begin{array}{c}
\tilde{A}_{\mathrm{L}} \\
\tilde{A}
\end{array}\right],
$$

where the two blocks are generated as follows:

$$
\begin{gathered}
\left(\tilde{A}_{\mathrm{L}}\right)_{i j}=\left.\mathrm{L} \varphi\left(\left\|\mathbf{x}-\xi_{\mathrm{j}}\right\|\right)\right|_{\mathbf{x}=\mathbf{x}_{\mathrm{i}}}, \quad \mathbf{x}_{\mathrm{i}} \in \mathcal{I}, \xi_{\mathrm{j}} \in \boldsymbol{\Xi}, \\
\tilde{A}_{i j}=\mathrm{B} \varphi\left(\left\|\mathbf{x}-\xi_{\mathrm{j}}\right\|\right), \quad \mathbf{x}_{\mathrm{i}} \in \mathcal{B}, \xi_{\mathrm{j}} \in \boldsymbol{\Xi} .
\end{gathered}
$$

The set of collocation points $\boldsymbol{\Xi}$ is split into a set of interior points $\mathcal{I}$ and a set of boundary points $\mathcal{B}$. The problem is well-posed if the linear system $\mathbf{A} \boldsymbol{\alpha}=\mathbf{b}$, with b as a vector consisting of entries $f\left(\mathbf{x}_{i}\right), \mathbf{x}_{i} \in \mathcal{I}$, followed by $g\left(\mathbf{x}_{i}\right), \mathbf{x}_{i} \in \mathcal{B}$, has a unique solution.

\subsection{RBF-based pseudospectral method}

The following review of non-symmetric RBF-based PS method is adapted from standard textbooks on Meshfree Approximation Methods with MATLAB [11]. An important feature of pseudospectral methods is the fact that one usually is content with obtaining an approximation to the solution on discrete set of grid points $x_{i}, i=1, \ldots, N$. One way to implement the spectral method is via so-called differentiation matrices $D$. These can be found so that the following equation holds 
at the collocation points

$$
\mathbf{u}^{\prime}=D \mathbf{u},
$$

where $\mathbf{u}=\left[\hat{u}\left(x_{1}\right), \ldots, \hat{u}\left(x_{N}\right)\right]$ is the vector of values of the approximation solution $\hat{u}$ at the collocation points. Usually, orthogonal polynomials such as Chebyshev polynomials are used as basis functions $B_{j}(x)$. In our case, we used MQ RBF.

The approximate solution is expressed as:

$$
\hat{u}(x)=\sum_{j=1}^{N} \beta_{j} B_{j}(x), \quad x \in \mathbb{R},
$$

If we evaluate (13) at the collocation points $x_{i}, i=1, \ldots, N$, we get

$$
\hat{u}\left(x_{i}\right)=\sum_{j=1}^{N} \beta_{j} B_{j}\left(x_{i}\right), \quad i=1, \ldots, N,
$$

or in matrix-vector notation

$$
\mathbf{u}=\mathbf{A} \boldsymbol{\beta}
$$

where $A_{i j}=B_{j}\left(x_{i}\right)$.

We can do the same in the case of derivative of $\hat{u}$ :

$$
\frac{d}{d x} \hat{u}(x)=\sum_{j=1}^{N} \beta_{j} \frac{d}{d x} B_{j}(x) .
$$

If we evaluate again at the collocation points $x_{i}$, then we get in matrix-vector notation

$$
\mathbf{u}^{\prime}=\mathbf{A}_{x} \boldsymbol{\beta}
$$

We can use (15) to formally solve for the coefficient vector $\boldsymbol{\beta}=\mathbf{A}^{-1} \mathbf{u}$ and rewrite eqn. (17) as

$$
\mathbf{u}^{\prime}=\mathbf{A}_{x} \mathbf{A}^{-1} \mathbf{u}
$$

so that the differentiation matrices $\mathbf{D}$ corresponding to (12) is given by

$$
\mathbf{D}=\mathbf{A}_{x} \mathbf{A}^{-1}
$$

For more complex linear differential operators $\mathcal{L}$ with constant coefficients we proceed in an analogous fashion to obtain differentiation matrix:

$$
\mathbf{L}=\mathbf{A}_{\mathcal{L}} \mathbf{A}^{-1}
$$

where the matrix $\mathbf{A}_{\mathcal{L}}$ has entries $\left(A_{\mathcal{L}}\right)_{i j}=\left.\mathcal{L} \varphi\left(\left\|\mathbf{x}-\xi_{j}\right\|\right)\right|_{\mathbf{x}=\mathbf{x}_{i}}$. We can use Kansa's non-symmetric method to obtain the discretized differential operator. The RBF 
collocation solution is obtained by solving the linear system

$$
\left[\begin{array}{c}
\tilde{A}_{\mathcal{L}} \\
\tilde{A}
\end{array}\right] \alpha=\left[\begin{array}{l}
\mathbf{f} \\
\mathbf{g}
\end{array}\right]
$$

where the matrices have the same form as in Kansa's approach. If we use $\alpha$ from (21) and once again assume the invertibility of the system matrix, we get

$$
\mathbf{u}=\mathbf{A} \boldsymbol{\alpha}=\mathbf{A}\left[\begin{array}{c}
\tilde{A}_{\mathcal{L}} \\
\tilde{A}
\end{array}\right]^{-1}\left[\begin{array}{l}
\mathbf{f} \\
\mathbf{g}
\end{array}\right]
$$

This suggests that the discretized differential operator $L$ based on the grid points $\mathbf{x}_{i}, i=1, \ldots, N$, and basis functions is given by

$$
\mathrm{L}_{\Gamma}=\left[\begin{array}{c}
\tilde{\mathbf{A}}_{\mathcal{L}} \\
\tilde{\mathbf{A}}
\end{array}\right] \mathbf{A}^{-1} \text {. }
$$

\subsection{Implicit discrete scheme}

We consider the implicit scheme of eqns. (1) and (3):

$$
\begin{gathered}
\varrho c_{p} \frac{T^{n+1}-T^{n}}{\Delta t}+k_{x} \frac{\partial^{2} T^{n+1}}{\partial x^{2}}+k_{y} \frac{\partial^{2} T^{n+1}}{\partial y^{2}}=0, \\
-k_{x} \frac{\partial T^{n+1}}{\partial x} e_{n x}-k_{y} \frac{\partial T^{n+1}}{\partial y} e_{n y}-h_{c}\left(T^{n+1}\right) \\
-\varepsilon_{R} \sigma\left(\left(T^{n+1}\right)^{4}-T_{R}^{4}\right)=-h_{c}\left(T_{A}\right),
\end{gathered}
$$

where $t_{n+1}=t_{n}+\triangle t, T^{n+1}$ and $T^{n}$ are the variable at time $t_{n+1}$ and $t_{n}$.

The approximate solution is expressed as:

$$
T\left(\mathbf{x}, t_{n+1}\right)=\sum_{j=1}^{N} \alpha_{j}^{n+1} \varphi_{j}(\mathbf{x}),
$$

where $\alpha_{j}^{n+1}, j=1, \ldots, N$, are the unknown coefficients to be determined and $\varphi_{j}(\mathbf{x})=\sqrt{\left(x-x_{j}\right)^{2}+\left(y-y_{j}\right)^{2}+c^{2}}$ are Hardy's multiquadrics functions [12].

By substituting eqn. (26) into eqns. (24) and (25) and using factorization for the radiation term $\left(T^{4}-T_{R}^{4}\right)$, we obtain:

$$
\begin{aligned}
\sum_{j=1}^{N}\left(\varrho c_{p} \frac{\varphi_{j}\left(\mathbf{x}_{i}\right)}{\triangle t}\right. & \left.+k_{x} \frac{\partial^{2} \varphi_{j}\left(\mathbf{x}_{i}\right)}{\partial x^{2}}+k_{y} \frac{\partial^{2} \varphi_{j}\left(\mathbf{x}_{i}\right)}{\partial y^{2}}\right) \alpha_{j}^{n+1} \\
& =\varrho c_{p} \frac{T^{n}\left(\mathbf{x}_{i}\right)}{\triangle t}, \quad i=1, \ldots, N-N_{\mathcal{B}}
\end{aligned}
$$




$$
\begin{array}{r}
\sum_{j=1}^{N}\left(-k_{x} \frac{\partial \varphi_{j}\left(\mathbf{x}_{i}\right)}{\partial x} e_{n x}-k_{y} \frac{\partial \varphi_{j}\left(\mathbf{x}_{i}\right)}{\partial y} e_{n y}-h_{c} \varphi_{j}\left(\mathbf{x}_{i}\right)\right. \\
\left.-\varepsilon_{R} \sigma\left(\varphi_{j}\left(\mathbf{x}_{i}\right)-T_{R}\right)\left(\varphi_{j}\left(\mathbf{x}_{i}\right)+T_{R}\right)\left(\varphi_{j}{ }^{2}\left(\mathbf{x}_{i}\right)+T_{R}^{2}\right)\right) \alpha_{j}^{n+1} \\
=-h_{c}\left(T_{A}\right), \quad i=N-N_{\mathcal{B}}+1, \ldots, N,
\end{array}
$$

where $N_{\mathcal{B}}$ and $N$ present the number at boundary and all discretized points.

The system of nonlinear equations which result from the space discretization of a nonlinear PDEs can be solved by Picard's or Newton's methods. In our case, we have used the Picard's method, also known as Successive Substitutions [13, 14].

\section{Numerical examples}

\subsection{One-dimensional charring}

The charring rate of wood usually refers to the dimensional rate, e.g. millimetres per minute, at which wood changes to char. Many factors are involved in wood charring. Kanury and Blackshear [15] examined various physical effects, including the diffusion of condensable vapours inward, internal convection outward, properties of the partially charred wood, kinetics of pyrolysis, energetic of pyrolysis, and postdecomposition reactions. No completely satisfactorily charring model has yet been developed.

An extensive data is available for simple one-dimensional charring. Therefore, a one-dimensional case of a timber slab of spruce, with a depth $d$, exposed to the standard fire [16] is analysed in order to compare the charring rate of the wood slab with the empirical models presented in the literature.

The charring of wood may be modelled by the mass loss rate $(\mathrm{g} / \mathrm{s})$ or by the rate of advance of the formed char front from the original surface $(\mathrm{mm} / \mathrm{min})$. Since the material properties at elevated temperatures are difficult to obtain, constant material properties of the wood and char are used. The following data has been used:

$$
\begin{aligned}
& T_{0}=20^{\circ} C, \varrho=370 \mathrm{~kg} / \mathrm{m}^{3}, k_{\text {wood }}=0.12 k_{\text {char }}=0.15 \mathrm{~W} / \mathrm{mK}, d=0.3 \mathrm{~m} \\
& h_{c}=22.5 \mathrm{~W} / \mathrm{m}^{2}, \epsilon_{R}=0.9, c_{p, \text { wood }}=1530 \mathrm{~J} / \mathrm{kgK}, c_{p, \text { char }}=1050 \mathrm{~J} / \mathrm{kgK} .
\end{aligned}
$$

Most known models suggest constant charring rates. In our case, we used a White and Norheim non-linear empirical model for charring rate of eight different wood species. The comparison to the present model in the case of spruce is shown in Fig. 1.

In all empirical models it is assumed that the charring of woods starts instantaneously after exposure to fire. In reality, this is not the case. In our model, the charring starts when the temperature of wood reaches the temperature of pyrolysis, which is around $300{ }^{\circ} \mathrm{C}$. This happens nearly 3 minutes after the fire starts. 


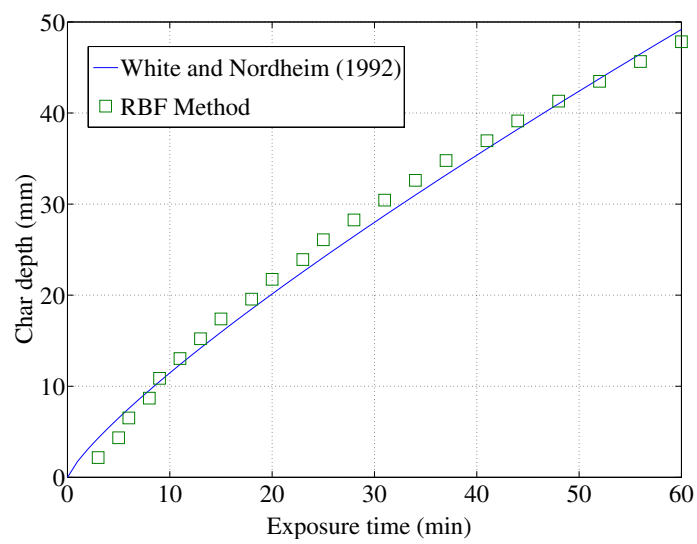

Figure 1: Comparison of White and Nordheim charring model to the present.

\subsection{A two-dimensional charring}

In the two-dimensional case, the formation of char in a timber beam exposed on three sides to the standard fire conditions in [16] is considered. The upper edge is thermally isolated. The original beam cross-section is rectangular with dimensions $10 \times 15 \mathrm{~cm}$. The beam cross-section is discretized by the mesh of $10 \times 10$ points. Material properties are assumed to be the same as the one-dimensional case. The results of the simulation at 10 and 30 minutes after the exposure to fire are given in figures 2 and 3 .
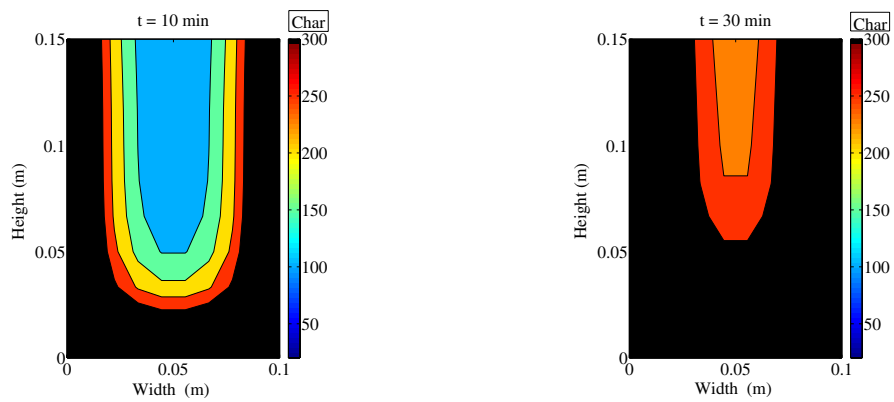

Figure 2: Temperature distribution in the cross-section of spruce beam and the transformation of wood into char at 10 and 30 minutes calculated with the Kansa approach, relaxation parameter: 0.14 . 

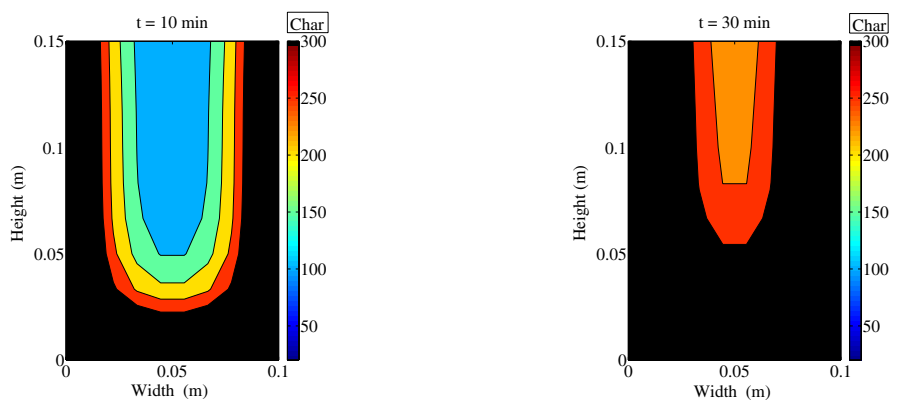

Figure 3: Temperature distribution in the cross-section calculated with the PS method, relaxation parameter: 0.14 .
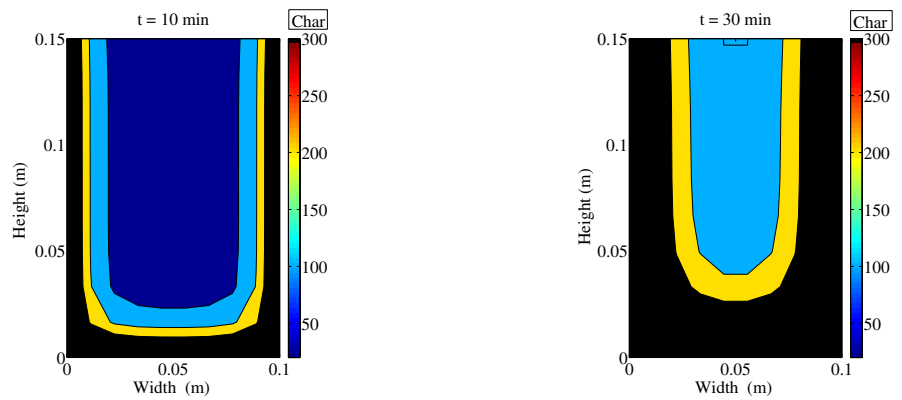

Figure 4: Temperature distribution in the cross-section calculated with the PS method, relaxation parameter: 0.1 .

\section{Conclusions}

Since the analytical solution is seldom obtainable, the idea of this paper was to represent an alternative approach to the conventional numerical methods. Kansa's and PS methods were presented. The results were tested on the one-dimensional case for which the comparison was made with the results obtained numerically and experimentally.

In Fig. 1, we can see that our approach gives the results which are comparable to the results obtained by the model proposed by White and Nordheim. The same model was used to analyze a two-dimensional behaviour of wood beam exposed to fire from three sides. It shows that the results are comparable to the results obtained in literature [6]. Comparison of the figure 2 and 3 indicates that MQ RBFs, PS, and Picard's methods give very similar results. Comparison of the results in figures 3 and 4 also show that the results are sensitive to the relaxation parameter. The simulations also show that badly scaled or narrow basis functions (e.g. linear $(\varphi(r)=r)$, cubic $\left(\varphi(r)=r^{3}\right)$, thin-plate spline $\left.\left(\varphi(r)=r^{2} \log r\right)\right)$ can prevent the effects of the boundary conditions from propagating inside the domain.

Therefore we can conclude that the presented methods could be an alternative to the conventional numerical methods. In our future work, moisture diffusion 
equations will be included and special attention should be given to the appropriate choice of RBFs.

\section{Acknowledgements}

The authors would like to thank the Slovenian Nuclear Safety Administration for their support. This work has been, in part, financed by the Slovenian Research Agency (ARRS) through the research program Geotechnology (PO-0268).

\section{References}

[1] Fredlund, B., Modelling of Heat and Mass Transfer in Wood Structures During Fire. Fire Safety Journal, 20, pp. 39-69, 1993.

[2] Kansa, E.J., Multiquadrics - A scattered data approximation scheme with applications to computational fluid-dynamics - Solution to parabolic, hyperbolic and elliptic partial differential equations. Comput. Math. Appl., 19, pp. 147-161, 1990.

[3] Forngerg, B., A Practical Guide to Pseudospectral Methods, Cambridge Univ. Press, 1998.

[4] Trefethen, L.N., Spectral Methods in MATLAB, SIAM (Philadelphia, PA), 2000.

[5] White, R.H. \& Nordheim, E.V., Charring rate of wood for ASTM E 119 exposure. Fire Technology, 28(1), pp. 5-30, 1992.

[6] Schnabl, S. \& Turk, G., Coupled heat and moisture transfer beams exposed to fire. WCTE 2006 - 9th World Conference on Timber Engineering - Portland, OR, USA, 2006.

[7] Luikov, A.V., Heat and Mass Transfer in Capillary-porous Bodies, Pergamon Press, Oxford, 1966.

[8] Wang, J.G. \& Liu, G.R., On the optimal shape parameters of radial basis functions used for 2-D meshless methods. Comput. Meth. Appl. Mech. Eng., 30, pp. 191-211, 2002.

[9] Vrankar, L., Turk, G. \& Runovc, F., Modelling of radionuclide migration through the geosphere with radial basis function method and geostatistics. Journal of the Chinese Institute of Engineers, 4/27, pp. 455-462, 2004.

[10] Vrankar, L., Kansa, E.J., Ling, L., Runovc, F. \& Turk, G. Moving-boundary problems solved by adaptive radial basis functions. Comput. Fluids, 39, pp. 1480-1490, 2010.

[11] Fasshauer, G.E., Meshfree Approximation Methods with MATLAB, Interdisciplinary Mathematical Sciences - 6, 2007.

[12] Hardy, R.L., Multiquadric equations of topography and other irregular surfaces. J. Geophys. Res., 176, pp. 1905-1915, 1971.

[13] Stephen, W. Goode, Differential Equations and Linear Algebra, 3rd edition, Prentice Hall, Upper Saddle River, NJ, Appendix 4, 2005. 
[14] Mai-Cao, L. Meshless Radial Basis Function Method for Unsteady Incompressible Viscous Flows. Ph.D. diss., University of Southern Queensland, Australia, 2009.

[15] Kanury, M.A. \& Blackshear, P.L. Jr., Some Considerations Pertaining to the Problem of Wood-Burning. Combustion Science and Technology, 1, pp. 339356, 1970.

[16] ISO 834, Fire-resistance test-Elements of building construction-Part 1. General requirements. ISO 834-1, International organization for standardization, Geneva, Switzerland, 1999. 